\title{
Adhesion Characteristics Analysis of Wafer Transfer Plane Based on the Acceleration Adjustment
}

\author{
Lei Shen ${ }^{1}$, Fang Song ${ }^{1,2}$, Yigang $\mathrm{Hu}^{1}$ and Tao $\mathrm{Chen}^{2}$ \\ ${ }^{1}$ Laboratory of Intelligent Control and Robotics, Shanghai University of Engineering Science, Shanghai, P.R. China; \\ ${ }^{2}$ Research Center of Robot and Micro system, Soochow University, SuZhou, Jiangsu, P.R. China
}

\begin{abstract}
As a key element of the semiconductor industry, efficient and stable transmission of the wafer has a very high demand for the wafer transfer manipulator. At present, the wafer transfer robots (SCARA-type, R- $\theta$ type) are widely used in IC manufacturing equipment. These transfer robots can only achieve the wafer movement along the plane and will not be able to change the attitude of the wafer movement during the wafer transmission. Due to the structure of the restrictions, the acceleration of the wafer transmission can only be achieved by the frictional force of the wafer itself in a vacuum environment. This transmission mechanism determines that it is difficult to further increase the acceleration of the wafer transmission and therefore it also affects the wafer transmission efficiency. Thus, from the microstructure adhesion mechanism, the attitude of the wafer transfer manipulator is proposed in this paper. The corresponding model is established and the acceleration characteristic is analyzed. It is of great theoretical significance to improve the efficiency of wafer transmission by this means.
\end{abstract}

Keywords: Wafer transfer, Pose adjustment, Acceleration, Microstructure.

\section{INTRODUCTION}

Integrated circuit (IC) equipment manufacturing equipment is a typical representative of the high technological equipment industry. Wafer transfer robot is one of the key equipments in the IC manufacturing equipment that is responsible for accurate positioning, and fast and steady transmission of wafer. The performance of the equipment directly affects the productivity and manufacturing quality of wafer production.

At present, there are two basic types of wafer transfer robots currently widely used in IC manufacturing equipment: flat joints type (SCARA type) and polar coordinates (R- $\theta$ type) (Fig. 1). However, these two systems can only achieve the wafer movement along the plane and will not be able to change the attitude of the wafer movement during the wafer transmission. Thus it is determined that adhesion between the end effector and the wafer can only rely on its own gravity to generate frictional force to achieve in vacuum environment. This transmission mechanism will limit the wafer transfer acceleration to improve further. Therefore, it has great significance to improve the transfer efficiency by way of adjusting the acceleration and the attitude of the manipulator to improve the transmission acceleration.

From the friction model, adhesion model and mechanism of the microstructure array, the adhesion characteristics and friction characteristics of the microarray based on the position adjustment of the manipulator are analyzed and the corresponding model is established.

*Address correspondence to this author at the School of Laboratory of Intelligent Control and Robotics, Shanghai University of Engineering Science, Shanghai, 201620, P.R. China; Tel: +86 021-67791247;

E-mail: songfang2005@163.com
Finally, the angle between the unit microstructure and the substrate and the maximum acceleration that can be allowed for the wafer transmission plane are determined based on the attitude adjustment. It is of great theoretical significance to improve the efficiency of wafer transmission by means of the acceleration adjustment and the attitude adjustment.

\section{ADHESION CHARACTERISTICS ANALYSIS OF WAFER TRANSFER PLANE}

The efficient and stable transmission of wafer puts forward higher requirements for the adhesion properties of the microstructure array: the high adhesion during the transfer process and the low adhesion during the desorption process. Therefore, based on the microstructure, the microarray is analyzed. The adhesion properties of the microarray with different tilt angles are analyzed.

When the microstructure is perpendicular to the substrate, the contact model of microstructure is shown in Fig. (2).

According to the theory of contact mechanics, the microadhesion force generates when the unit microstructure is in contact with the rigid plane is:

$$
\begin{aligned}
F_{f}= & \sqrt{6 \pi r^{3} K w_{f}} \\
K= & \frac{4}{3}\left(\frac{1-v_{s}^{2}}{E_{s}}+\frac{1-v_{f}^{2}}{E_{f}}\right)^{-1} \\
& r \text {---the radius of the microstructure, } \mathrm{mm} ; \\
& K \text {---the equivalent elastic modulus, } M P a ; \\
& w_{f} \text {---surface energy of the contact surface, } \mathrm{mJ} / \mathrm{m}^{2} ; \\
& v_{s} \text {---Poisson's ratio of the contact surface ; }
\end{aligned}
$$




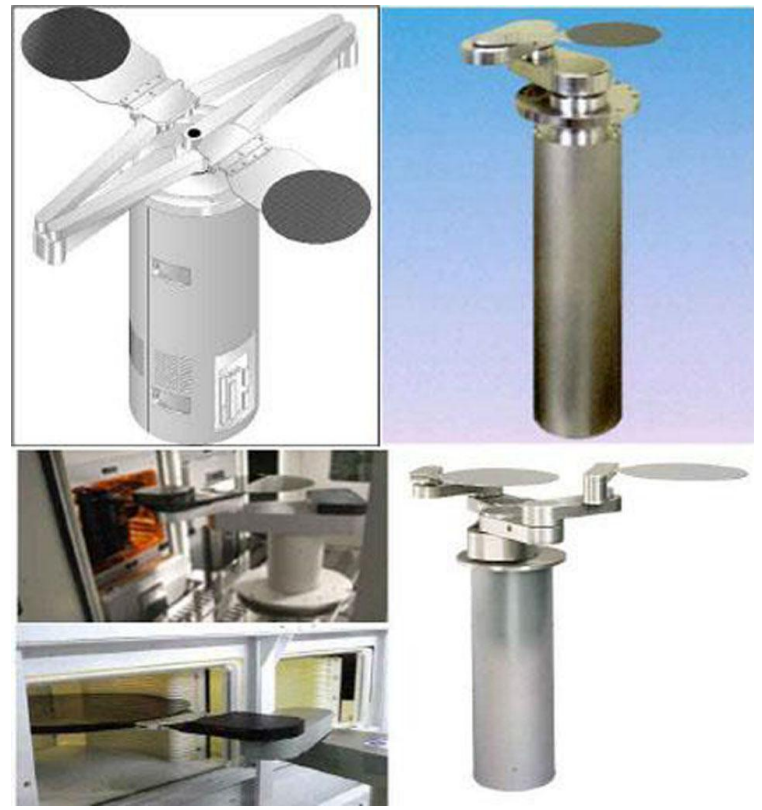

Fig. (1).Typical robots for wafer transmission.

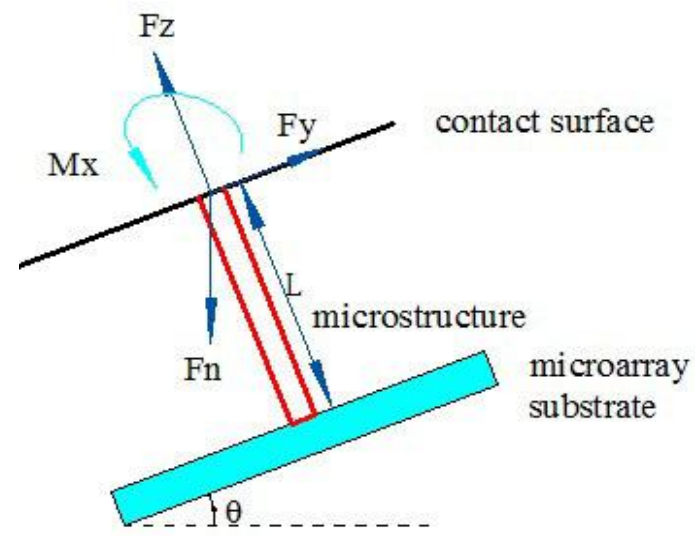

Fig. (2). Analysis diagram of adhesion when the microstructure is perpendicular to the substrate.

$v_{f^{--}}$Poisson's ratio of the microstructure ;

$E_{s}$---elastic modulus of contact surface material, GPa;

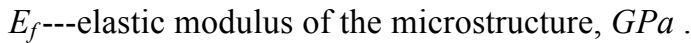

When microstructure produces adhesion force $F_{f}$, adhesive strength per unit area is generated by:

$\sigma_{c}=\frac{F_{f}}{\pi r^{2}}$

At this point, in order to simplify the analysis of adhesion, the unit microstructure is assumed to be flexible cylindrical beam model fixed in the substrate (shown in Fig. 2). In the adhesion process, one end of the microstructure in contact with the wafer does not reverse, but only move in the vertical direction. So the rotating torque of the microstructure end is:

$M_{x}=\frac{F_{y} L}{2}$
$F_{y^{---}}$the radial component of the cylinder, $F_{y}=F_{n} \sin \theta, N$;

$L$--- the length of the cylinder, $m m$;

$\theta$--- the rotation angle of the end effector, ${ }^{\circ}$.

According to the mechanics analysis of material, the existence of $M_{\mathrm{x}}$ makes the cylinder half of the pull, half of the compression. The tensile stress $\sigma_{1}$ caused by $M_{x}$ is:

$$
M_{x}=\frac{F_{n} \sin \theta L}{2}=\int_{A}(-y) \sigma_{1} d A=\sigma_{1} \frac{4}{3} r^{3}
$$

That is,

$\sigma_{1}=\frac{3 F_{n} L \sin \theta}{8 r^{3}}$

In addition, the presence of the axial component $F_{z}$ causes tensile stress in the cylinder, and the tensile stress $\sigma_{2}$ produced by $F_{z}$ is: 


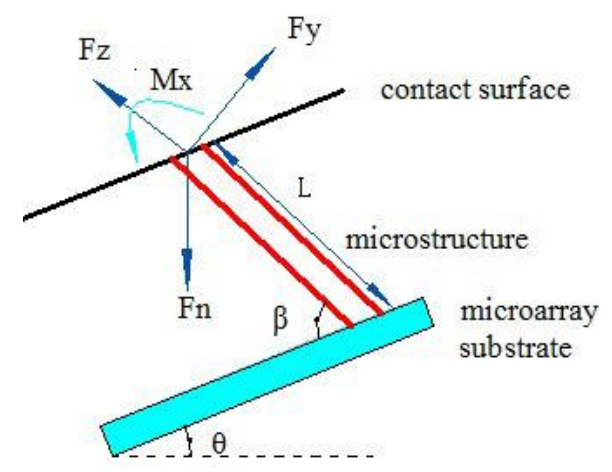

Fig. (3) Analysis diagram of adhesion when the microstructure and the substrate are at a certain slope angle.

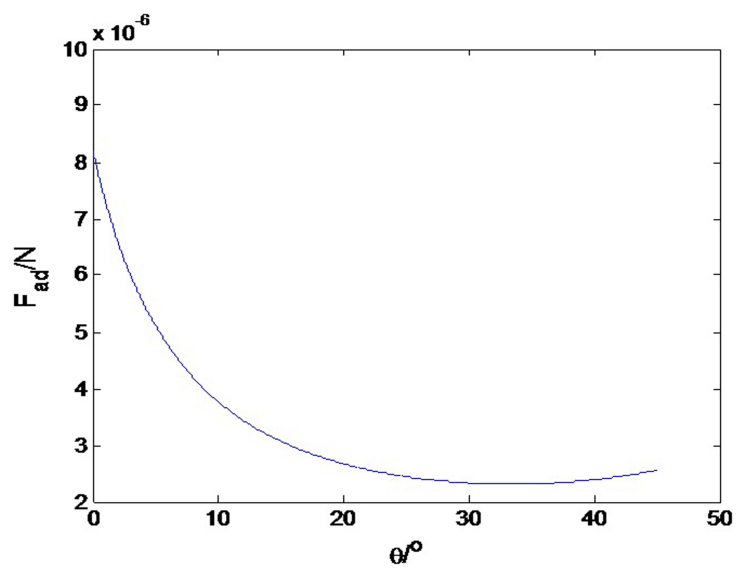

Fig. (4). The adhesion force with different $\theta$ when the microstructure is perpendicular to the substrate.

$\sigma_{2}=\frac{F_{n} \cos \theta}{\pi r^{2}}$

To sum up the above two effects, the average tensile stress generated in cross-section is:

$\sigma_{c}=\frac{3 F_{n} L \sin \theta \cos ^{2} \theta}{8 r^{3}}+\frac{F_{n} \cos \theta}{\pi r^{2}}$

For the microstructures under the position adjustment of the end effector, the maximum adhesion force is the force when the tensile stress $\sigma_{\mathrm{c}}$ reaches the adhesive strength. Therefore, when the microstructure is perpendicular to the substrate, the adhesive force of the microstructure in contact with the plane under different pose is:

$$
F_{a d}=\frac{8 r^{2} \sqrt{6 \pi r K w_{f}}}{\cos \theta(3 \pi L \sin \theta \cos \theta+8 r)}
$$

Similarly, when the microstructure and the substrate have a certain inclination angle, the force model of the microstructure is shown in Fig. (3).

Then, adhesion between the microstructure and the plane under different pose is:

$$
F_{a d}=\frac{8 r^{2} \sqrt{6 \pi r K w_{f}}}{\cos \left(\frac{\pi}{2}+\theta-\beta\right)\left[3 \pi L \sin \left(\frac{\pi}{2}+\theta-\beta\right) \cos \left(\frac{\pi}{2}+\theta-\beta\right)+8 r^{2}\right]}
$$

When the microstructure is perpendicular to the substrate, take a kind of typical microstructure material, its structural parameters is: $K=5.0 \mathrm{MPa}, w_{f}=45.72 \mathrm{~mJ} / \mathrm{m}^{2}, \quad L=15 \mathrm{um}$, $r=2.5 \mathrm{um}$, and substitute these parameters into the formula for calculating the adhesion. The relationship between the adhesion force with the change of pose angle can be obtained, as is shown in Fig. (4).

When the wafer is transmitted by means of acceleration regulation, the transmission process is achieved by constantly adjusting the pitch angle of the end effector to adjust the acceleration. In this process, it has a large adhesion force to be advantaged to the steady transmission of the wafer. At the beginning and end of the transfer of the desorption process, the end of the actuator is in a horizontal pose state, then it is easy to desorp with a small adhesion.

\section{ACCELERATION PERFORMANCE ANALYSIS BASED ON POSE ADJUSTMENT}

When the microstructure is perpendicular to the substrate, the mechanics model of the maximum acceleration that the end effector can provide at different pitch angles is shown in Fig. (5).

When the wafer is transported in vacuum environment, the friction between the microstructure array and the wafer is:

$f=\hat{u} F_{n}=\hat{u} m g \cos \theta$ 


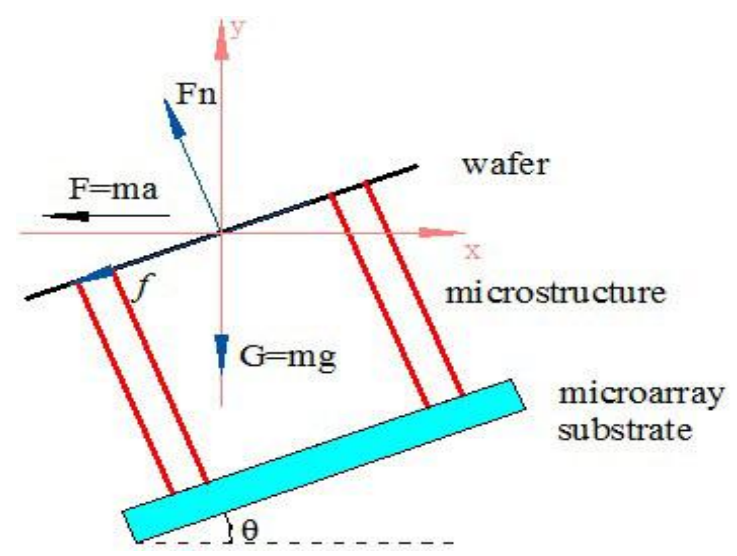

Fig. (5). Analysis diagram of acceleration when the microstructure is perpendicular to the substrate.

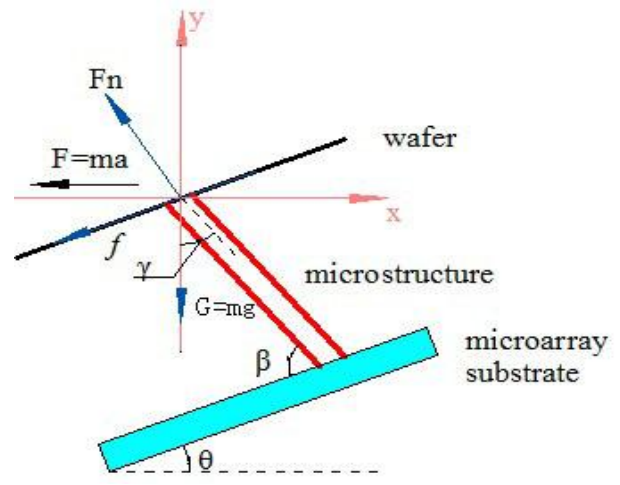

Fig. (6). Analysis diagram of acceleration when the microstructure and the substrate are at a certain slope angle.

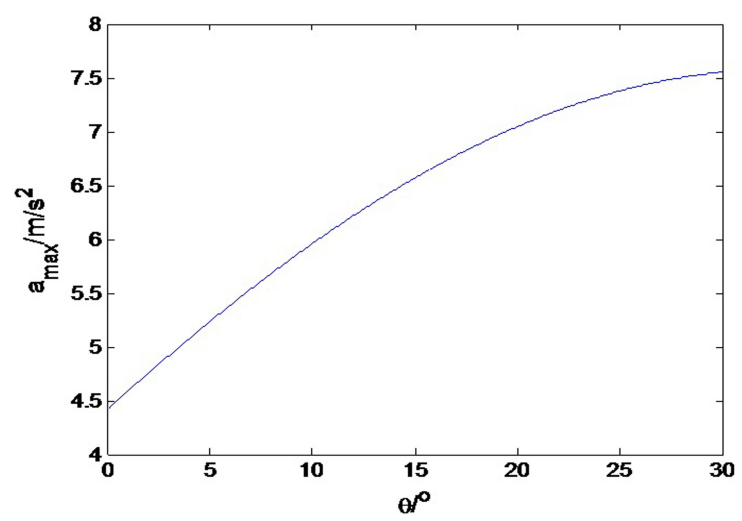

Fig. (7). Acceleration with different $\theta$ when the microstructure is perpendicular to the substrate.

According to the contact characteristics analysis of the microstructure array, the following formula can be obtained:

$\hat{u}=u+\tau A_{f} / F_{c r}$

$F_{c r}=\frac{\pi^{2} E I}{(\alpha L)^{2}}, I=\frac{\pi r^{4}}{4}$

$\tau$--- the shear strength between the wafer and the microarray, $\mathrm{Pa}$;

$A_{f}$---the actual contact area between the microstructure and the wafer, $m^{2}$; $N$;

$F_{c r}$--- the critical force of the microstructure instability, $I$--- the microstructure's sectional moment of inertia , $m^{4}$; $E$--- the microstructure's elastic modulus, $\mathrm{Pa}$.

According to Newton's law, the maximum acceleration that can be allowed by the end effector to transmit the wafer by pose adjustment is:

$a_{\max }=g \cos \theta \sin \theta+\left(\mu+\frac{16 \tau L^{2}}{\pi^{2} E r^{2}}\right) g \cos ^{2} \theta$ 


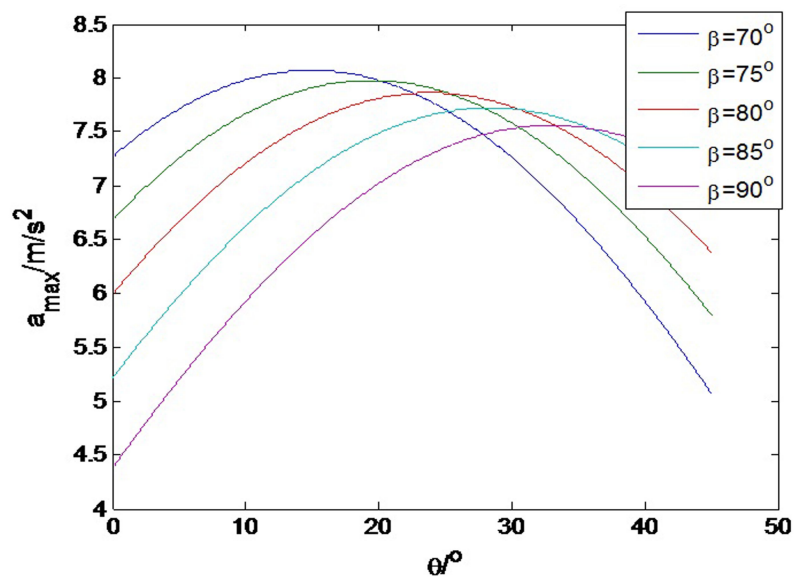

Fig. (8). Acceleration with different $\theta$ when $\beta$ is at a certain slope angle.

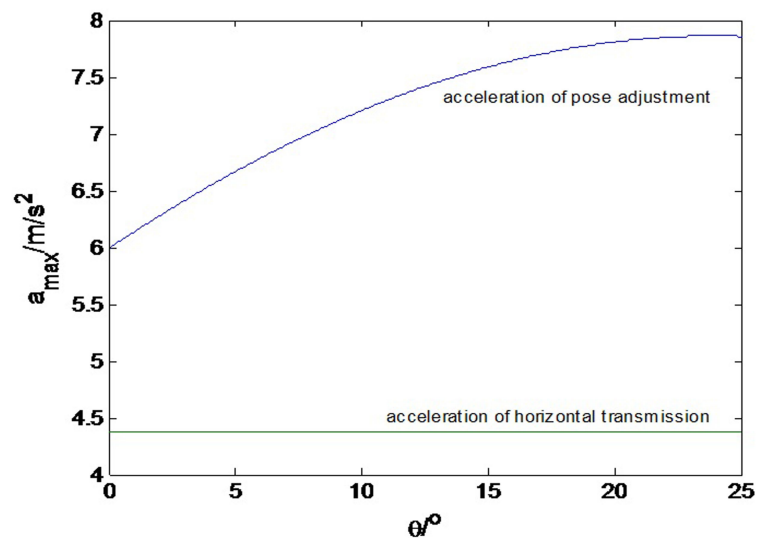

Fig. (9). Comparative analysis diagram of acceleration.

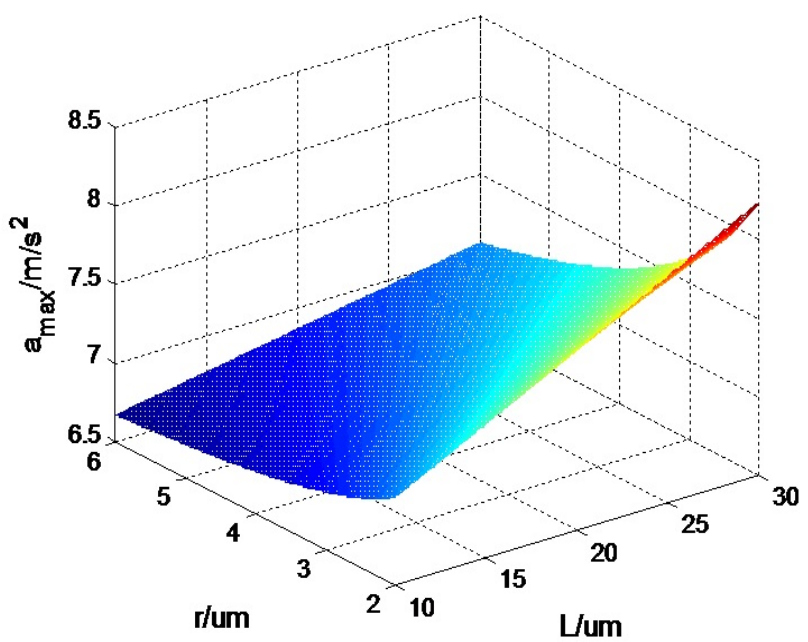

Fig. (10). Analysis diagram of acceleration with different $r$ and $L$.

Similarly, when the microstructure and the substrate show a certain tilt angle, the force model of acceleration analysis is shown in Fig. (6).

Now, the maximum acceleration that the end effector can permit is: $a_{\max }=g \sin \gamma \cos \gamma+\left(u+\frac{16 \tau L^{2}}{\pi^{2} E r^{2}}\right) g \cos \gamma \cos \theta$

When the microstructure is perpendicular to the substrate, take a kind of typical microstructure material, whose struc- 
tural parameters are: $g=9.8 \mathrm{~m} / \mathrm{s}^{2}, \tau=10 M P a, L=15 \mathrm{um}$, $E=3.5 G P a, r=2.5 u m, u=0.28$. The relationship between the maximum acceleration with the change of pose angle can be obtained, as is shown in Fig. (7).

Similarly, when the microstructure and the substrate are at a certain tilt angle, the following conclusions can be obtained: when the angle $\beta$ between the microstructure and the substrate is constant, the curve between the maximum acceleration $a_{\max }$ with the change of pose angle $\theta$ is shown in Fig. (8).

By the above analysis on the adhesion and acceleration, it can be seen that when using the way of pose adjustment to transfer wafer and the tilt angle of the microstructure is $80^{\circ}$, the microarray has small adhesion. However, it can provide much larger acceleration than the horizontal pose transmission, as is shown in Fig. (9).

In addition, based on the above analysis of micro interfacial adhesion mechanism, it can be seen that when the end effector is at a certain pose angle, the maximum acceleration change with the radius $r$ and the length $L$ of the microstructure is shown in Fig. (10).

\section{CONCLUSION}

In this paper, by analyzing the adhesion mechanism of the microstructure array, the adhesion force and the acceleration force models under different pose angles are established. In addition, the relationship between the acceleration and the radius and length of the microstructure is explored. This analysis is of great theoretical significance to improve the efficiency of the wafer transmission by means of acceleration regulation and manipulator pose adjustment.

\section{CONFLICT OF INTEREST}

The authors declared that they have no conflicts of interest to this work.

\section{ACKNOWLEDGEMENTS}

It is a project supported by External-Planned Task (NO. SKLRS-2014-MS-10) of State Key Laboratory of Robotics and System (HIT) \& Jiangsu Provincial Key Laboratory of Advanced Robotics Fund Projects (JAR201401).

\section{REFERENCES}

[1] C. Bonora, and R. G. Hine, "Ultra low contact area end effector", US Patent No. 2006/0181095A1, 2006.

[2] M. Cong, Y. Du, and B. Shen, "Robotic wafer handling systems for integrated circuit manufacturing: a review", Robot, vol. 29, no. 3, pp. 261-6, 2007

[3] W. Gan, "Research on kernel technology of a parallel wafer robot", Master's thesis, Tianjin University, Tianjin, pp.9-11, 2007.

[4] . Lee, C. Majidi, B. Schubert, and R. S. Fearing, "Sliding induced adhesion of stiff polymer microfiber arrays: 1 . Macroscale behaviour", Journal of Royal Society Interface, vol. 5, no. 25, pp. 835844, 2008.

[5] W. Tang, W. F. Chow, and W. L. Yi, "End effector for transferring a wafer", US Patent No. 2007/0177963 A1, 2007.

[6] K. L. Johnson, K. Kendall, and A. D. Roberts, "Surface energy and the contact of elastic solids", Proceedings of the Royal Society of London, vol. A324, pp. 301-13, 1971.

[7] M. Quirk, J. Serda, Z. S. Han, Semiconductor Fabrication Technology. Publishing House of Electronics Industry, Beijing, pp. 4$15,2002$.

[8] S. C. Hu, Y. K. Chuah, and M. C. Yen, "Design and evaluation of a minienvironment for semiconductor manufactures", Building and environment, vol. 37, pp. 201-208, 2002.

Received: July 01, 2015

Revised: August 10,2015

Accepted: August 30, 2015

(C) Shen et al.; Licensee Bentham Open.

This is an open access article licensed under the terms of the Creative Commons Attribution Non-Commercial License (http://creativecommons.org/licenses/by$\mathrm{nc} / 4.0 /$ ) which permits unrestricted, non-commercial use, distribution and reproduction in any medium, provided the work is properly cited. 